\title{
Information Technology - the key enabler of multiple project management in higher education institutions
}

\author{
Raluca VASILACHE \\ "Dunarea de Jos" University, Galati, Romania \\ raluca.vasilache@ugal.ro \\ Alexandru CAPATINA \\ "Dunarea de Jos" University, Galati, Romania \\ alexandru.capatana@ugal.ro \\ George Cristian SCHIN \\ "Dunarea de Jos" University, Galati, Romania \\ george.schin@ugal.ro
}

\begin{abstract}
The body of knowledge related to the impact of IT on project management (PM) acknowledges the driving and restraining forces for PM software implementation. However, the peculiarities of such kind of approach have received little research attention at the level of Romanian public institutions. This paper addresses this research gap in the attempt to outline how PM software becomes the key enabler of solution to level the resources involved in multiple projects managed in the same time by a public institution from higher education system. The methodological approach involves a case study, which highlight the value of IT support in the process of identifying and leveling the overallocated resources within multiple project management.
\end{abstract}

Keywords: multiple project management, software capability, higher education, resource allocation, resource pool.

\section{Introduction}

Existing body of knowledge regarding Project Management (PM) software implementations in the higher education institutions reveals important challenges and opportunities in managing simultaneous projects.

The simultaneous management of multiple projects has been often analyzed by both researchers and practitioners. It has been suggested that up to $90 \%$ of all projects occur in the multi-project context (Payne, 1995).The issues of complexity, capacity, conflict, commitment, which are specific to simultaneous project management context can be alleviated, to some extent, by integrating project plans where common project management pillars are implemented, such a shared resource pool and independent reporting systems.

Higher education institutions must be permanently aware of the risks that can occur while they manage multiple projects. A research conducted by Raz and Michael (2001) emphasize the main tools having the greatest potential for contribution to a multiple project risk management process: risk impact assessment, cost-benefit analysis during risk planning, cause and effect analysis during risk mitigation plan.

Although many studies acknowledge the benefits of PM software in managing simultaneous projects, the peculiarities of such kind of approach have received little research attention in Romania. 
The main objective of this study is to identify the key capabilities of PM software correlating with success in managing multiple projects in higher education institutions. In comparison to previous research, the qualitative approach of this challenging objective aims to provide more generalizable results on how higher education institutions can mitigate the risks while implementing multiple projects.

The present research challenges the assumptions that the implementation of PM software is a necessary condition for capturing opportunities to effectively manage multiple projects, able to foresight post-implementation users' acceptance, while being involved in simultaneous projects.

\section{Literature review}

The very first issue related to multiple project management relies on the additional workload imposed on the management, as this context involves multiple smaller projects to be dealt with rather than a single large project. The coordination team of simultaneous projects must spend more time monitoring the situation, as there are more risks than in the particular case of a single project (Payne, 1995).

Theoretical implications on multi-project environment research have a limited empirical foundation; most of the contributions are based on the researchers' practical experience or on findings from a specific field or organization. There is little knowledge concerning which factors are context-specific to simultaneous project management. In this context, this research proposes to close a gap in what concerns the peculiarities of this multi-project approach in a higher education institution.

In a simultaneous project management approach, the projects are implemented by allocating resources from a common resource pool. It means that the projects should be integrated into the management control and reporting system of the organization that embraces this approach (Van Der Merwe, 1997). Multi-project management has to go beyond resource allocation and propose incentive structures, accounting systems, instead of more scheduling, progress reports, or more time spent on review meetings (Engwall and Jerbrant, 2003).

The management approach of a multi-project environment differs in a great extent by the single project environment, as management efforts and activities that focus on group of projects are considerably higher (McDonough III and Spital, 2003). Reviewing the simultaneous projects as a big organizational project strongly correlates with success, while predetermined rules and procedures seems to have no correlation with success in the case of multiple project management (Dietrich and Lehtonen, 2005).

The main challenges associated with the goal of achieving the most effective output from managing multiple projects have been outlined by three building blocks: alignment management; control and communication; learning and knowledge management. A common problem encountered by organizations dealing with a portfolio of projects is the difficulty of maintaining control and communication (Dooley et al., 2005).

Higher education institutions have traditionally been characterized by knowledgesharing cultures, which make it likely that collaborative work patterns are still prevalent in the way simultaneous projects are managed. As academic institutions increasingly adopt multiple projects in order to cope with the increasing demands the research poses, a better 
understanding of the multi-project management benefits is essential for learning how to operate and develop the project team members' skills (Van Ameijde et al., 2009).

The current challenge for policymakers from higher education institutions is to acknowledge and accept that there have been significant changes in the methods to manage multiple projects and the establishment of a single point of simultaneous projects support becomes critical (Garrison and Kanuka, 2004).

PICBE |1004

The intensive use of specific methods and information technology tools lead to superior performances in multi-project management. A survey of 753 project managers was conducted to rate several project management tools. Respondents were asked to rate tools based on their potential to enable project success. The most effective tools for multiproject environments are considered those that had high use and high potential for improving project success, including software for task scheduling, multiple projects requirements analyses, progress reports, and alerts for change requests (Schwalbe, 2015).

PM software packages are positioned as either high-end or low-end, depending on the price that organizations are willing to pay. Only high-end solutions have the capability to handle large projects and perform simultaneous project management tasks. A survey conducted by Liberatore and Pollack-Johnson (2003) highlight that PM software is mainly used for planning (95\%), and for control (80\%) in the context of a multi-project environment.

Perceived usefulness, ease of use and friendly interface are considered the PM software drivers, able to mediate the effect of internal pressures on resources involved in simultaneous projects, and on behavioral intention to adopt a specific technology (Davis and Venkatesh, 2004).

Project professionals are ready to accept the implementation of project management tools primarily after preliminary tests on the quality of their outputs. The second driving force appears to be project complexity, as they tend to use the PM software when dealing large projects (Ali et al., 2008). Multi-project scenarios require shared PM software features, able to purposely address the project managers' needs.

\section{Methodology}

This research involves a qualitative approach - a case study, based on a focus-group with 20 project managers from a higher education institution from Romania, who accepted the challenge to present their own perceptions regarding the role of PM software in managing simultaneous projects.

The participants to the focus group were able to bring to the fore ideas and experiences in relation with PM software capabilities in five "hot" issues related to multiple project management (Figure 1). 


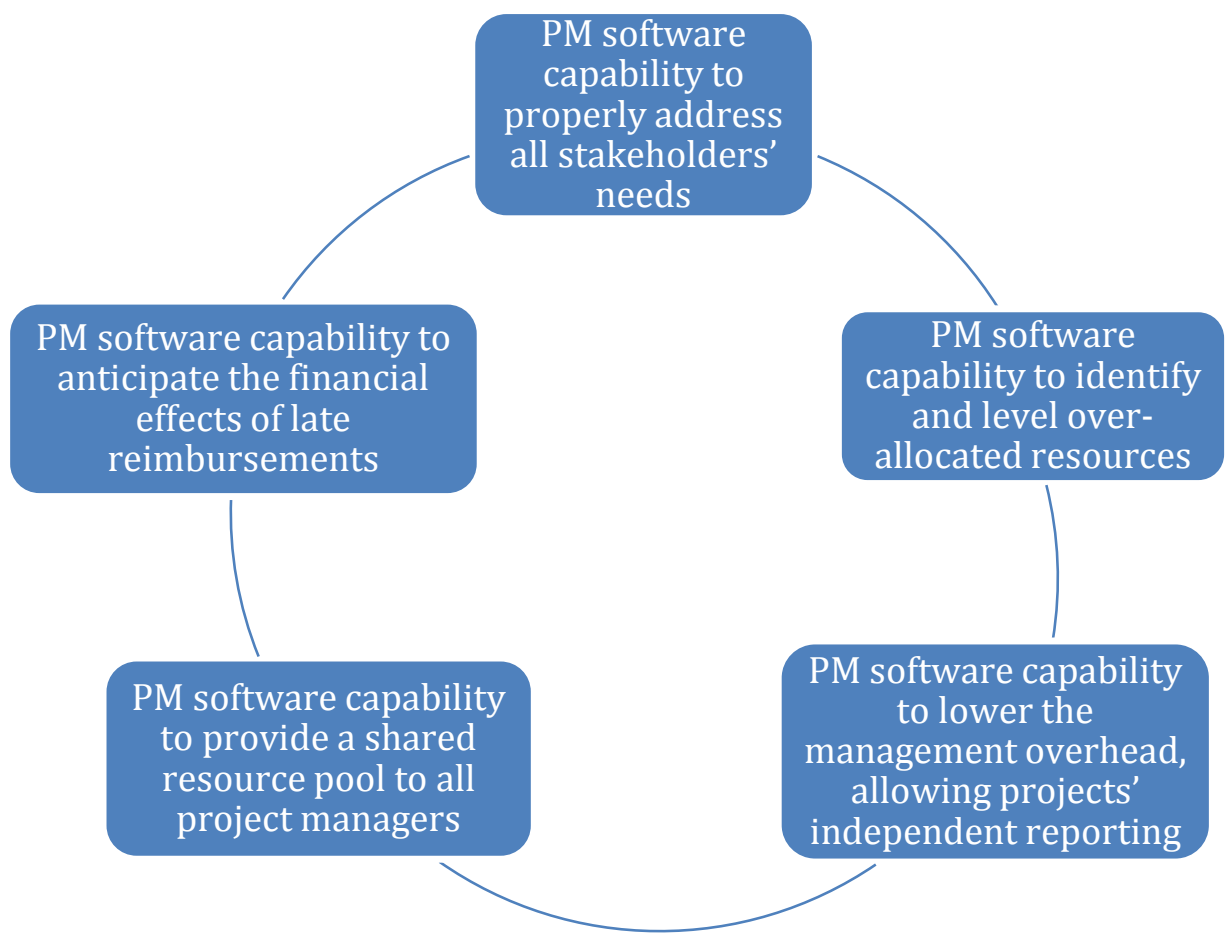

Figure 1 - Research conceptual framework

Source: Authors' own research.

The focus group technique provides the facilitators (research team members) the opportunity to analyze the ways in which project managers collectively make sense of the five construct meanings around the conceptual model.

We have tailored the research design in order to create a storytelling context, where project managers highlighted their perceptions on the way PM software helped them provide with the insights needed for research objectives. Their experiences the storylines needed to understand the level they assigned to PM software capabilities in relation with the five hot issues embedded into the conceptual framework.

\section{Results and discussions}

The key to effectively manage multiple projects in a higher education institution is to understand the information technology roles in dealing with a wide range of situations regarding the resource allocation, contingency plans and unexpected legislative changes that might occur.

The stakeholders involved in the process of managing multiple projects within a higher education institution are project managers as well as their teams, specialized staff from the department responsible with research and operational projects management, the Rector, the partners, the project target groups and the management authorities which receive the reimbursement orders.

Most of the project managers' answers reveal a high PM software capability to properly address all stakeholders' needs, which create the framework for resources 
assignment in multiple projects to the highest priority projects, in order to be completed on time, within budget, and to the assumed output indicators (Figure 2).

\section{PM software capability to properly address stakeholders' needs while managing multiple projects}

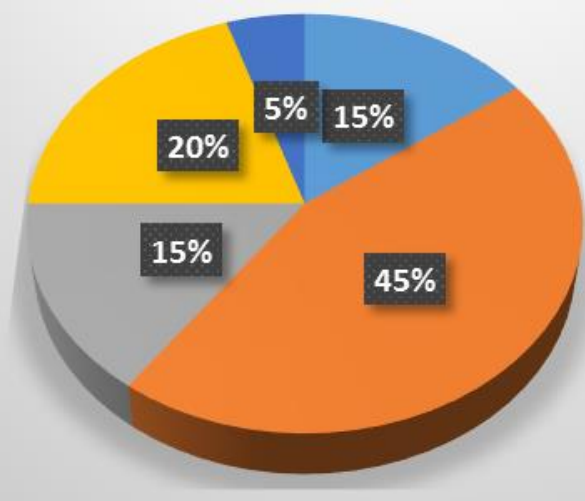

Very high

- High

Medium

Low

Very low

\section{Figure 2 - PM software capability to properly address all stakeholders' needs}

Source: Authors' own research.

Allocating resources, especially human and financial resources, to multiple projects managed in the same time by a higher education institution represents a difficult task. The more specific the skills and knowledge required for each project are, especially in research projects, the more difficult the entire allocation process is.

The feature regarding the identification and leveling the over-allocated resources should be regarded as compulsory for any PM software solution which can be integrated in the IT infrastructure of an academic institution. In general, human resources tend to be over-allocated in multiple projects managed in the same time by a higher education institution, as the human capital is the most important asset in research and operational projects. The respondents involved in the current case study are fully aware of the necessity to embed such a capability in the PM software, according to their answers (Figure 3). 


\section{PM software capability to identify and level the over-allocated resources within multiple project \\ management}

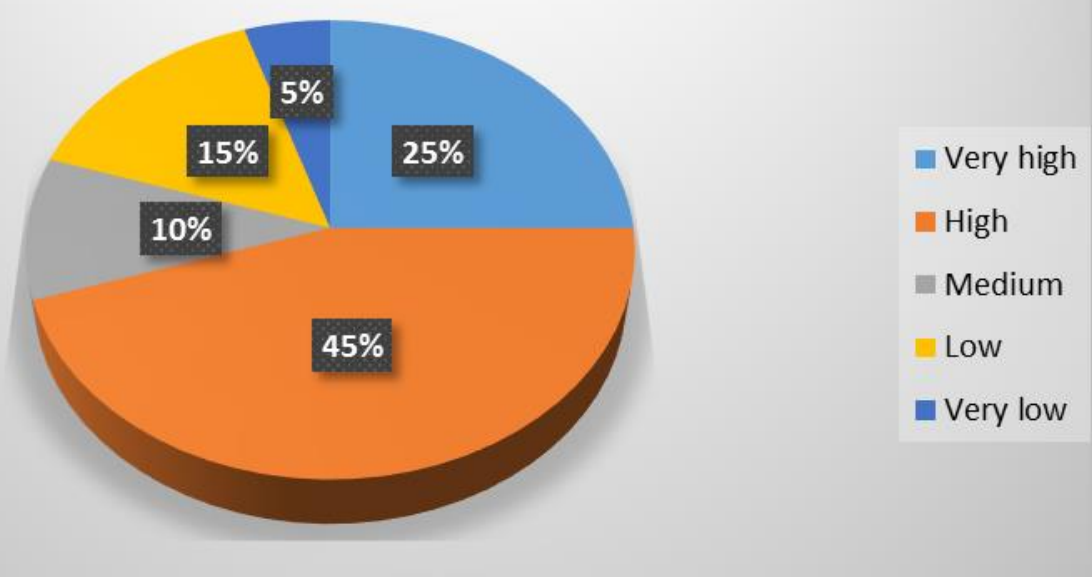

PICBE $\mid 1007$

\section{Figure 3 - PM software capability to identify and level over-allocated resources}

Source: Authors' own research.

The resource over-allocation syndrome in multi-project environments is frequently found in academic institutions, due to project managers' propensity to select the highlyskilled persons to successfully meet their projects' goals, without considering their involvement in concurrent projects.

Allowing independent reporting represents a risk mitigation initiative developed by project managers involved in a multi-project academic environment, aiming to lower the top management overhead in the context of multi-tasking approach of projects.

Respecting the milestones of major phases within a group of projects managed simultaneously by a higher education institution will also enable project managers and especially their teams to balance their workload and involvement within each project. Managing common resources' allocation across different projects allows project managers to avoid over committing shared resources across multiple projects. The answers received within the case study highlight PM software high capability (45\%), respectively very high capability (25\%) to lower the management overhead by allowing an independent reporting (Figure 4).

One of the main advantages of consolidating the financial reporting of separate projects into one single financial database is the ability to integrate financial data within simultaneous projects' work break down structures, enabling task dependency that might appear between more interdependent projects. 


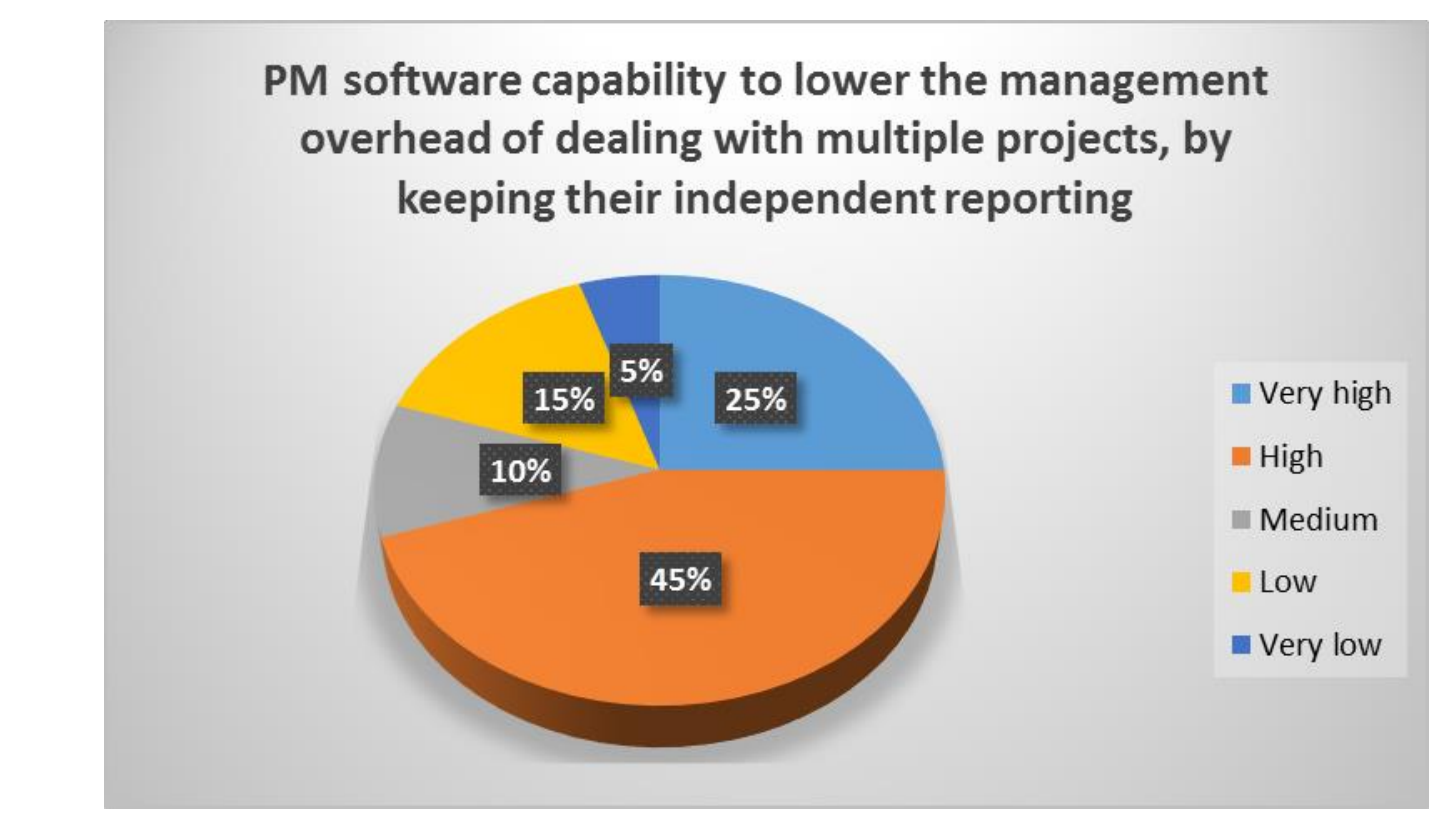

PICBE $\mid 1008$

\section{Figure 4 - PM software capability to lower the management overhead, allowing projects' independent reporting}

Source: Authors' own research.

It is common for simultaneous projects implemented within academic institutions to share human, equipment and material resources. Setting up a resource pool by the department responsible of managing projects enhances resource management effectiveness, especially when several projects share the same resources. This approach could be accomplished only if a resource-sharing academic culture has been established and Share Resources features are available in PM software.

Once a resource pool is designed and shared between multiple projects, project managers can easily detect any resource assignment made within a project, emphasized on the academic resource sharing platform and highly visible to all other projects involved in this collaborative approach of a shared resource pool. Figure 5 outlines the respondents' largest perception to consider the medium PM software capability to provide a shared resource pool to all project managers (30\%). We expected to see a higher perception of resource-sharing academic culture, but it seems this is not the case in this research sample. 


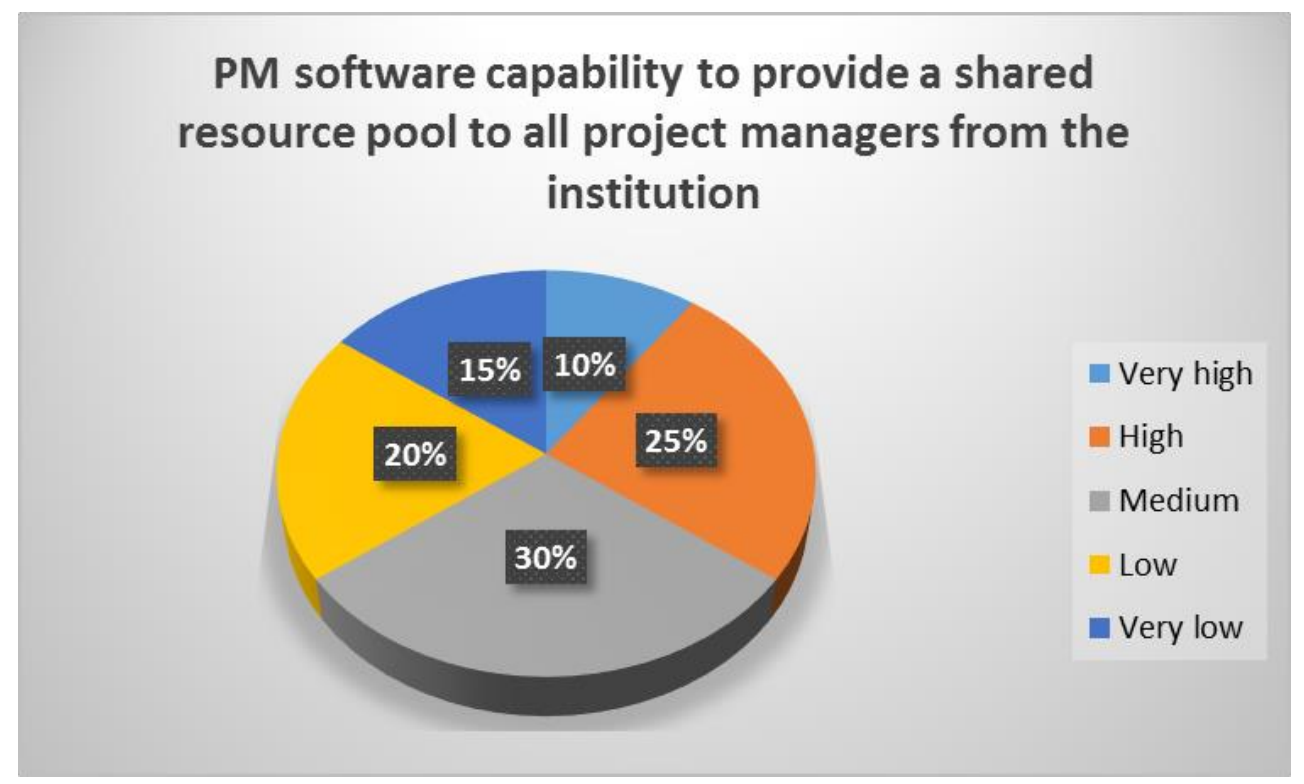

PICBE |1009

\section{Figure 5 - PM software capability to provide a shared resource pool to all project managers}

Source: Authors' own research.

Project managers are fully aware that late reimbursements of their requests lead in many cases to projects' delays if contingency plans are not properly prepared and the institution hasn't the capacity to provide financial support to minimize the risks of late payments made by management authorities. The capability to detect early warnings regarding reimbursement delays makes the difference between the PM software available on the market. Higher education institutions should pay a great attention to the existence of this feature when they launch acquisition process of PM software. The capacity to anticipate weak signals related to late payments allows a balanced distribution of institutional financial resources among projects in progress.

Respondents perceive that this kind of capability (Figure 6) is extremely important for a "healthy" financial allocation for the simultaneous projects management by a higher education institution. 


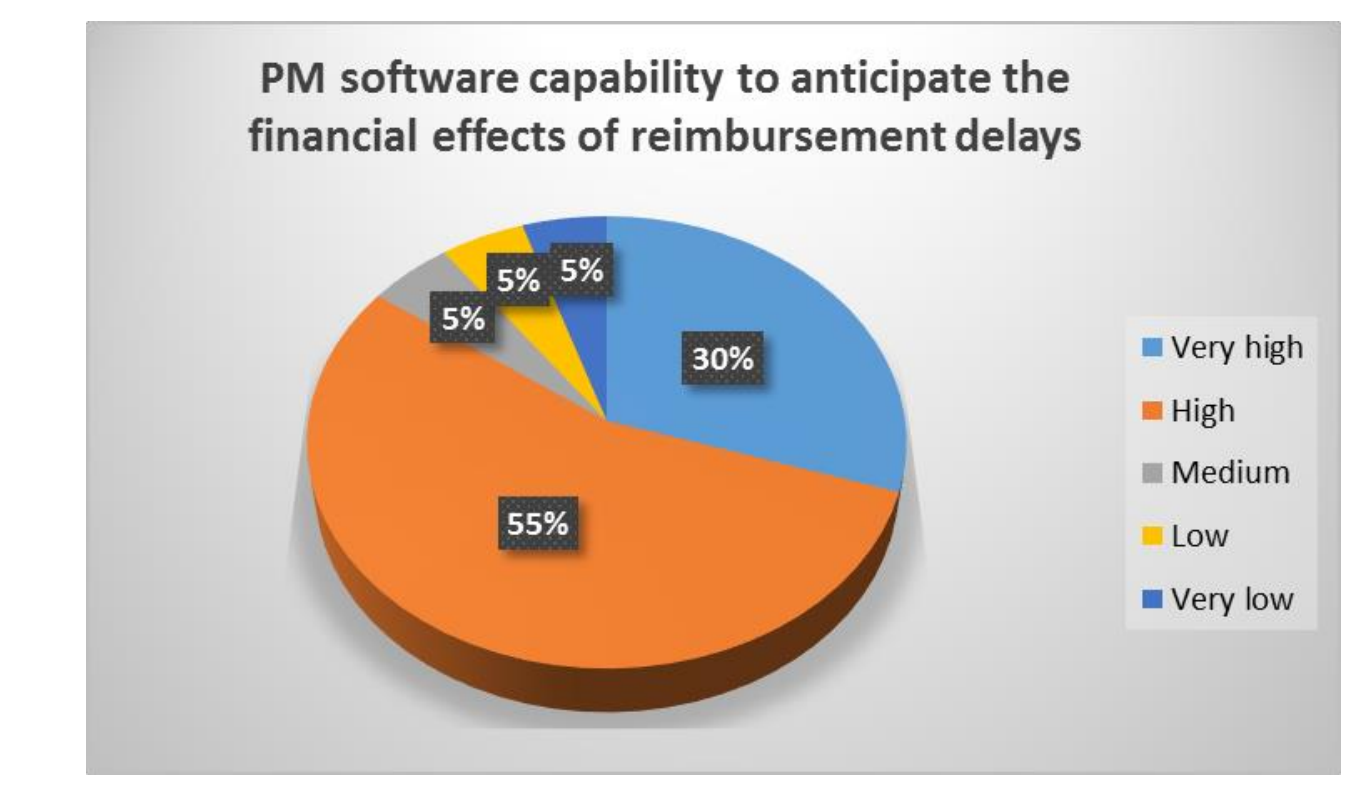

PICBE $\mid \mathbf{1 0 1 0}$

\section{Figure 6 - PM software capability to anticipate the financial effects of late reimbursements}

Source: Authors' own research.

Latest generation of PM software are endowed with early warning alerts in view to minimize potential risks associated with late payments from management authorities. Project managers need to fully understand how to benefit from these early warning alerts and make decisions accordingly.

\section{Conclusions}

Most of the findings of this study are in line with prior studies dealing with PM software benefits from the body of knowledge in managing multi-project contexts. Our attempt was to shed some light on the assumptions behind methodological approaches to PM benefits in managing simultaneous projects within higher education institutions.

This research results provide some compelling and interesting insights for institutional governance in universities, as their staff is frequently involved in simultaneous projects. Moreover, the outcomes of current research purposefully address on-going gaps in the PM body of knowledge regarding how to effectively manage multiple projects by means of IT solutions within academic sector.

In our opinion, the results can be helpful in broadening the boundaries of the PM software implications in simultaneous project management body and contributing to more effective resource allocation in multi-project environments. When Project managers are confronted with difficult resource allocation decisions in the context of competing projects, PM software could provide relevant outputs to the process of prioritizing the resource assignment in simultaneous projects.

The present study outlines that higher education institutions' expectations about a PM software, captured using reliable qualitative data, are more predictable comparing to those that would have been obtained using quantitative measures. 
The main limitation of the study consists in the small number of project managers from one university that participated in the focus group, but the sample could be extended in the future, after targeted discussions with projects managers from other Romanian universities.

This study provides useful insights that can be further used to evaluate the predictors of PM software effectiveness in higher education institutions, characterized by the need of managing simultaneous projects.

We intend to develop this research by means of qualitative comparative analyses based on deliverables from the simultaneous projects managed in a three years period, which will contribute to the identification of new meanings and causal configurations of conditions leading to the improvement of multi-project scenarios in public universities.

\section{References}

Ali, A. S. B., Anbari, F. T., \& Money, W. H. (2008). Impact of organizational and project factors on acceptance and usage of project management software and perceived project success. Project Management Journal, 39(2), 5-33.

Davis, F. D., \& Venkatesh, V. (2004). Toward preprototype user acceptance testing of new information systems: implications for software project management. IEEE Transactions on Engineering Management, 51(1), 31-46.

Dietrich, P., \& Lehtonen, P. (2005). Successful management of strategic intentions through multiple projects-Reflections from empirical study. International Journal of Project Management, 23(5), 386-391.

Dooley, L., Lupton, G., \& O'Sullivan, D. (2005). Multiple project management: a modern competitive necessity. Journal of Manufacturing Technology Management, 16(5), 466-482.

Engwall, M., \& Jerbrant, A. (2003). The resource allocation syndrome: the prime challenge of multi-project management?. International Journal of Project Management, 21(6), 403-409.

Garrison, D. R., \& Kanuka, H. (2004). Blended learning: Uncovering its transformative potential in higher education. The Internet and Higher Education, 7(2), 95-105.

Liberatore, M. J., \& Pollack-Johnson, B. (2003). Factors influencing the usage and selection of project management software. IEEE transactions on Engineering Management, $50(2), 164-174$.

McDonough III, E. F., \&Spital, F. C. (2003). Managing project portfolios. ResearchTechnology Management, 46(3), 40-46.

Payne, J. H. (1995). Management of multiple simultaneous projects: a state-of-the-art review. International Journal of Project Management, 13(3), 163-168.

Raz, T., \& Michael, E. (2001). Use and benefits of tools for project risk management. International Journal of Project Management, 19(1), 9-17.

Schwalbe, K. (2015). Information technology project management. Cengage Learning.

Van Ameijde, J. D., Nelson, P. C., Billsberry, J., \& Van Meurs, N. (2009). Improving leadership in higher education institutions: A distributed perspective. Higher Education, 58(6), 763.

Van Der Merwe, A. P. (1997). Multi-project management-organizational structure and control. International Journal of Project Management, 15(4), 223-233. 